\title{
Evidencia Convergente y Discriminante del TAI-Estado: Influencia de las Estrategias de Afrontamiento Pre-examen
}

\author{
Evidence of Convergent and Discriminant Validity of TAI-State: Influence \\ of Pre-exam Coping Strategies
}

Evidência convergente e discriminante do TAI-Estado: Influência de estratégias de enfrentamento da ansiedade pré-testes

Sergio Dominguez-Lara* http://www.orcid.org/0000-0002-2083-4278

Instituto de Investigación de Psicología, Facultad de Ciencias de la Comunicación, Turismo y Psicología, Universidad de San Martín de Porres, Lima, Perú.

$\nabla$

Recibido: 19-06-17 Revisado: 15-11-17 Aceptado: 15-03-18 Publicado: 20-12-18

- Resumen. El objetivo fue conocer cuánto influyen las estrategias de afrontamiento preexamen (EAPE) sobre la Ansiedad ante Exámenes - Estado (AE-Estado) en estudiantes de psicología para obtener evidencias convergentes y discriminantes del TAI-Estado. En el estudio participaron 156 estudiantes de psicología (115 mujeres) de una universidad privada de Lima Metropolitana, con edades entre18 y 42 años $(M=22.51)$. Los instrumentos usados fueron: el Test Anxiety Inventory - Estado (TAI-Estado) y el Coping with PreExam Anxiety and Uncertainty-Breve (COPEAU-B), que evalúa cuatro estrategias. Fue utilizado un análisis factorial confirmatorio para verificar la confiablidad y validez, y modelos de ecuaciones estructurales para evaluar el modelo de investigación. Luego del análisis de regresión estructural, las EAPE explican el 13.3\% de la varianza de la AEEstado, siendo las estrategias orientación a la tarea y búsqueda de apoyo social por razones instrumentales las de mayor influencia $(B=-.212$ y $B=.320$, respectivamente). Se discuten las implicancias teóricas y prácticas del estudio.
Palabras clave: ansiedad ante exámenes, afrontamiento pre-examen, estudiantes universitarios, validez, confiabilidad 
Abstract. The objective was to find out the degree to which the pre-exam coping strategies (PECS) influence the Test Anxiety (TA) - State (TA-State) in psychology studentsin order to obtain convergent and discriminating evidence from the TAI-State. The study included 156 psychology students (115 women) from a private university in Metropolitan Lima, ages ranging from 18 to $42(M=22.51)$. The instruments used were: Test Anxiety Inventory-State (TAI-State) and the Coping with Pre-Exam Anxiety and Uncertainty-Brief (COPEAU-B), which evaluates four strategies. A confirmatory factor analysis was used to verify reliability and validity; while structural equation models were used to assess the research model. After the structural regression analysis, the PECSs explain 13.3\% of the variance of the TA-State, with task-oriented and search for social support for instrumental reasons strategies as the most influential $(B=-.212$ and $B=.320$ ). The theoretical and practical implications of the study are discussed.

- Resumo. O objetivo foi conhecer atéque ponto influem as estratégias deenfrentamento pré-teste (EEPT) sobre a Ansiedade diante de Exames - Estado (AE-Estado) em estudantes de psicologia para obter evidências convergentes e discriminantes do TAI-Estado. Participaram no estudo 156 estudantes de psicologia (115 mulheres) de uma universidade privada de Lima Metropolitana, com idades de entre 18 e 42 anos $(\mathrm{M}=22.51)$. Os instrumentos utilizados foram: o Test Anxiety Inventory - Estado (TAIEstado) e o Coping with Pre-Exam Anxiety and Uncertainty-Breve (COPEAU-B), que avalia quatro estratégias. Utilizou-se uma análise fatorial confirmatória para verificar a confiabilidade e validade, e modelos de equações estruturais para avaliar o tipo de pesquisa. Após a análise de regressão estrutural, as EAPE explicam o 13,3\% da variância da $A E$-Estado, onde as estratégias orientação à tarefa e busca de apoio social por razões instrumentais são as de maior influência ( $\beta=-.212$ e $\beta=.320$, respectivamente). Discutem-se as implicações teóricas e práticas do estudo.
Keywords:

test anxiety, pre-exam coping, university students, validity, reliability

Palavras-chave: ansiedade diante de testes, enfrentamento pré-teste, estudantes universitários, validade, confiabilidade

$\mathrm{n}$ el contexto de la educación superior universitaria, las evaluaciones cumplen un rol preponderante debido a que permiten al docente tener un panorama sobre el aprendizaje de los estudiantes. Aunado a ello, son situaciones que provocan en los estudiantes diversas reacciones, siendo una de las más frecuentes la ansiedad. En este sentido, la ansiedad ante exámenes (AE) hace referencia a un rasgo de personalidad específico para exhibir estados de ansiedad de forma más intensa y frecuente de lo habitual, con preocupaciones que interfieren con la atención, concentración y realización de exámenes 
(Spielberger, 1980; Spielberger \& Vagg, 1995). Esta definición se basa en una experiencia constante, es decir, considerando a la AE como un rasgo de personalidad (AE-Rasgo) aunque específico de situaciones evaluativas, pero sin circunscribir dicha experiencia a un examen en concreto. Sin embargo, y pese a su importancia, la AE experimentada en el examen en sí mismo (AE-Estado) ha sido parcialmente ignorada.

La AE-Estado se define como un periodo de ansiedad transitorio provocado por una situación de examen específica (Hong \& Karstersson, 2002), y es influida de forma dinámica por factores personales (e.g., ansiedad como rasgo) y situacionales (e.g., asignatura) (Spielberger \& Vagg, 1995), llegándose incluso a postular que la AE-Rasgo es potenciada por diferentes factores situacionales (Zohar, 1998). Asimismo, diversos estudios la han asociado inversamente con la atención selectiva y la concentración (Fernández-Castillo \& Caurcel, 2015), memoria de trabajo (Ikeda, Iwanaga \& Seiwa, 1996), y el rendimiento en exámenes (Dominguez-Lara, 2017a; Dominguez-Lara, Calderón-De la Cruz, Alarcón-Parco, \& Navarro-Loli, 2017), dado que esta faceta de la AE tiende a incrementarse ante la proximidad de un examen, haciendo más fuerte su relación negativa con la autoeficacia académica (Lotz \& Sparfeldt, 2017; Roick \& Ringeisen, 2017), por lo que es esperada la asociación negativa con el rendimiento.

A pesar de estos hallazgos, los estudios en habla hispana que operacionalizan el constructo AE-Estado en un instrumento son recientes. El único antecedente se focaliza en la adaptación de la versión en español del Test Anxiety Inventory (TAI) a la situación de evaluación (TAI-Estado; Dominguez-Lara, 2016a), mientras que en habla inglesa existen otra versión adaptada del TAI (Hong, 1988), adjetivos (Lotz \& Sparfeldt, 2017; Roick \& Ringeisen, 2017), o enfocar las respuestas de la sección Estado del Inventario de Ansiedad Rasgo-Estado (Zohar, 1998), para evaluar la AE-Estado.

El estudio de la estructura interna del TAI-Estado pudo dilucidar algunos aspectos relacionados con la configuración del instrumento. Para comenzar, la estructura de dos factores (preocupación y emocionalidad) brindada en el modelo que subyace a las diversas versiones del TAI no fue respaldada, obteniendo un solo factor denominado AE-Estado. Además, los indicadores de confiabilidad de las puntuaciones y de constructo fueron elevados (>.90), tal como fue observado en el estudio estructural del TAI en universitarios peruanos (Dominguez-Lara \& De la Cruz-Contreras, 2017). Aunque la evidencia obtenida respecto de la estructura interna fue favorable, debe ser complementada con otras fuentes de validez para consolidar el instrumento, sobre todo con relación a la asociación de la AE-Estado con constructos afines, denominada en forma más reciente como evidencias de validez con relación a otras variables, la cual incluye la evidencia convergente/discriminante que hace referencia a la asociación con otras medidas que reflejen constructos teóricamente relevantes o relaciones test-criterio, donde se vincula las puntuaciones del instrumento con una medida de desempeño (AERA, APA \& NCME, 2014).

En este sentido, y si bien los hallazgos preliminares de su relación con el rendimiento en exámenes (Dominguez-Lara, 2017a; Dominguez-Lara et al., 2017) es evidencia con respecto a un 
criterio (AERA, APA \& NCME, 2014), es necesaria su asociación con constructos relevantes a su red nomológica (Taras \& Kline, 2010). Entonces, las estrategias de afrontamiento pre-examen (EAPE) son una opción adecuada debido a su asociación con la AE-Rasgo.

Las EAPE pueden definirse como una serie de estrategias utilizadas por los estudiantes días antes del examen y que podrían ubicarse en la fase anticipatoria de afrontamiento (Carver \& Scheier, 1994), y comprende tanto la orientación a la tarea (OT), evitación (E) y búsqueda de apoyo social (BAS), que a su vez se divide en BAS por razones emocionales y BAS por razones instrumentales (Dominguez-Lara \& Merino-Soto, 2016).

Con respecto a estudios conjuntos de la AE y las EAPE, por un lado han sido halladas relaciones moderadas y positivas entre la AE-Rasgo con las estrategias BAS y OT, y bajas o nulas con la estrategia E (Piemontesi \& Heredia, 2011; Stöber, 2004); mientras que otro estudio indica relaciones bivariadas negativas con la OT, y positivas con BAS y E, y los resultados de su análisis multivariado indican que BAS y E contribuían de forma significativa a la variabilidad de la AERasgo (Putwain, Connors, Symes \& Douglas-Osborn, 2012).

A la fecha, no han sido hallados estudios que analicen el patrón correlacional de las EAPE y la AE-Estado, se espera que las magnitudes de dichas correlaciones no difieran de lo hallado en estudios preliminares que evalúan la relación entre las EAPE y la AE-Rasgo, reflejando los patrones conductuales esperados de los estudiantes evaluados. Además, al establecerse en un plano temporal distinto, es decir, que las EAPE aparecen antes que la AE-Estado, se hipotetiza una influencia significativa de las EAPE sobre la experiencia de ansiedad durante el desarrollo del examen (AE-Estado).

Por ello, el objetivo del presente estudio fue obtener evidencia convergente/discriminante del TAI-Estado mediante el análisis de la influencia de las EAPE sobre la AE-Estado bajo un enfoque de modelos estructurales. Las hipótesis de estudio fueron:

- $\mathrm{H}_{1}$ : Existirá una influencia negativa y significativa de la estrategia OT sobre la AE-Estado.

- $\mathrm{H}_{2}$ : Existirá una influencia positiva y significativa de las estrategias de BAS sobre la AE-Estado.

- $\mathrm{H}_{3}$ : No existirá influencia significativa de la estrategia E sobre la AE-Estado.

Estos planteamientos tienen coherencia, ya que si la persona utiliza estrategias que optimicen el tiempo de estudio (e.g., OT) y que relacionan con la autoeficacia académica (Dominguez-Lara, 2018) sentiría mayor confianza durante la evaluación $\left(\mathrm{H}_{1}\right)$ que el estudiante que basó su estrategia en solicitar consejo a sus condiscípulos sobre la forma de abordar determinado examen (e.g., BAS) $\left(\mathrm{H}_{2}\right)$. Sin embargo, evitar estudiar los temas involucrados al examen (estrategia E) no muestra una relación consistente con la AE-Estado, por lo que en la presenta investigación se hipotetiza que no contribuye a la variabilidad de esta última $\left(\mathrm{H}_{3}\right)$.

Estos resultados servirán para conocer una faceta no explorada con respecto a la relación en la AE-Estado con las EAPE utilizadas en el contexto peruano, tanto con fines 
instrumentales como empíricos. Los hallazgos proveerán evidencias de validez adicionales que permitirán usar el TAI-Estado en el contexto de educación superior, y desde lo empírico, ayudarán a la toma de decisiones para potenciar o modificar aquellas estrategias que tengan un impacto mayor sobre la experiencia de ansiedad durante el examen, debido a la relación de esta última con el rendimiento académico.

\section{MÉTODO}

\section{Participantes}

En este estudio instrumental basado en una estrategia asociativa (Ato, López, \& Benavente, 2013) participó una muestra intencional formada por 156 estudiantes universitarios (115 mujeres; 2 no consignaron el dato) de quinto $(n=57)$, sétimo ciclo $(n=37)$ y octavo ciclo $(n=62)$ de la carrera profesional de psicología de una universidad privada ubicada en Lima Metropolitana. Las edades estuvieron comprendidas entre los 18 y 42 años (86.8\% entre 18 y 25 años; 4 no consignaron la edad) $\left(M_{\text {edad }}=22.51, D E_{\text {edad }}=3.42\right)$, y con diferencias moderadas a favor de los hombres $\left(t_{[148]}=2.874, p=.005, d=.53\right)$.

\section{Instrumentos}

Test Anxiety Inventory - Estado (TAI-Estado). El TAI-Estado (Dominguez-Lara, 2016a) evalúa la ansiedad ante exámenes como constructo unidimensional y está compuesto por 15 ítems con cuatro alternativas de respuesta (Nada, Algo, Bastante y Mucho). La interpretación de los puntajes es directa: a mayor puntaje, mayor ansiedad durante el examen. A fin de focalizar al respondiente en la situación de examen, todos los enunciados están antecedidos por la frase “Durante el examen...”.

Coping with Pre-Exam Anxiety and Uncertainty-Breve (COPEAU-B). Fue empleada la versión breve realizada en universitarios peruanos (Dominguez-Lara \& Merino-Soto, 2017) formada por 12 ítems con seis opciones de respuesta (desde Nunca hasta Siempre). El COPEAU-B evalúa cada estrategia con tres ítems: Orientación a la Tarea y Preparación, Búsqueda de Apoyo Social por razones emocionales, Búsqueda de Apoyo Social por razones instrumentales, y Evitación. La interpretación de los puntajes es directa: a mayor puntaje, mayor frecuencia de uso de la estrategia evaluada.

\section{Procedimiento}

Los estudiantes fueron informados acerca de los objetivos del estudio, indicándoles que su participación será voluntaria, y que no serán acreedores dealgún tipo derecompensa académica o económica y que las respuestas que brinden serán de carácter confidencial. Al término, se les 
agradeció por su colaboración. Los instrumentos fueron aplicados inmediatamente después de rendir el examen final indicándoles que respondan el TAI-Estado en base a cómo se sintieron durante el examen. Asimismo, respecto al COPEAU-B se indicó que lo respondan de forma específica pensando en la asignatura. Esto garantizaría que las respuestas brindadas a ambos cuestionarios estén relacionadas con la misma asignatura.

Análisis preliminar. Ante la imposibilidad de analizar la invarianza de medición entre varones y mujeres debido al tamaño muestral de los grupos (Dimitrov, 2010) fueron comparados sus coeficientes a como un indicador aproximado de equivalencia entre estos (Merino, \& Lautenschlager, 2003). Para tal fin, fue utilizado el método de Feldt y Kim (2006) con el programa LittleAlpha (Merino-Soto, 2016). En vista de las características del presente estudio, la elección de este método se justifica sobre otros porque está orientado a muestras pequeñas $(n<100)$ o a instrumentos con pocos ítems $(1<k<4)$. Posteriormente, se reportaron los estadísticos descriptivos (media y desviación estándar) y distribucionales (asimetría y curtosis) de cada ítem, así como la normalidad univariada (por cada dimensión) usando la prueba de ShapiroWilk (SW; 1965; Ghasemi \& Zahediasl, 2012).

Análisis de modelos de medición. Previamente fue analizada la normalidad multivariada mediante el coeficiente de Mardia, esperando magnitudes adecuadas (< 70; Rodríguez \& Ruiz, 2008). Posteriormente fueron considerados dos modelos. El primero trata de un modelo unidimensional $\left(\mathrm{M}_{1}\right)$, es decir, donde todos los ítems son influidos simultáneamente por un factor. El segundo modelo $\left(\mathrm{M}_{2}\right)$ se trata de cinco factores oblicuos (cuatro dimensiones del COPEAU-B y una del TAI-Estado). El método de estimación fue el de máxima verosimilitud robusto con corrección por no-normalidad (Satorra \& Bentler, 1994), y la matriz utilizada fue la de correlaciones policóricas (Lee, Poon \& Bentler, 1995) debido al exceso de curtosis de algunos ítems. Los modelos fueron valorados según los índices de ajuste tradicionales (CFI > .95, RMSEA < .05 y SRMR < .05). Con respecto a ello, se espera que $\mathrm{M}_{1}$ tenga un ajuste pobre, a modo de línea base, ya que de lo contrario indicaría que los cinco factores analizados representan facetas de un solo constructo. En consecuencia, se espera que $M_{2}$ tenga significativamente un mejor ajuste que $M_{1}$.

Adicionalmente fueron reportadas las cargas factoriales, la varianza extraída promedio por factor (AVE; Fornell, \& Larcker, 1981) y las correlaciones inter-factoriales ( $\phi$ ) con el objetivo de proveer evidencias de validez interna convergente. En este sentido, las magnitudes del AVE superiores a .50 y coeficientes de confiabilidad por encima de .60 (Hair, Black, Babin, Anderson \& Tatham, 2010) son indicadores de adecuada validez convergente. Complementariamente, para el análisis de la validez interna discriminante fueron comparados el coeficiente de determinación factorial $\left(\phi^{2}\right)$ y la AVE, esperando que el segundo sea de mayor magnitud. Finalmente, fueron reportados dos coeficientes de confiabilidad a nivel de variables latentes: el $\omega$ (McDonald, 1999) y el $H$ (Hancock \& Mueller, 2001). Todos esos coeficientes fueron calculados 
con base al modelo de medición oblicuo. No fueron reportados los coeficientes vinculados a las puntuaciones observadas (e.g., a) en estos análisis, ya que se trabajó con variables latentes.

Análisis del modelo estructural. Fue implementado un modelo de regresión estructural (Kline, 2016) a fin de analizar la influencia de las estrategias de afrontamiento pre-examen (variables exógenas) sobre la ansiedad ante los exámenes (variable endógena) ( $\mathrm{M}_{3}$ ) (ver Figura 1).

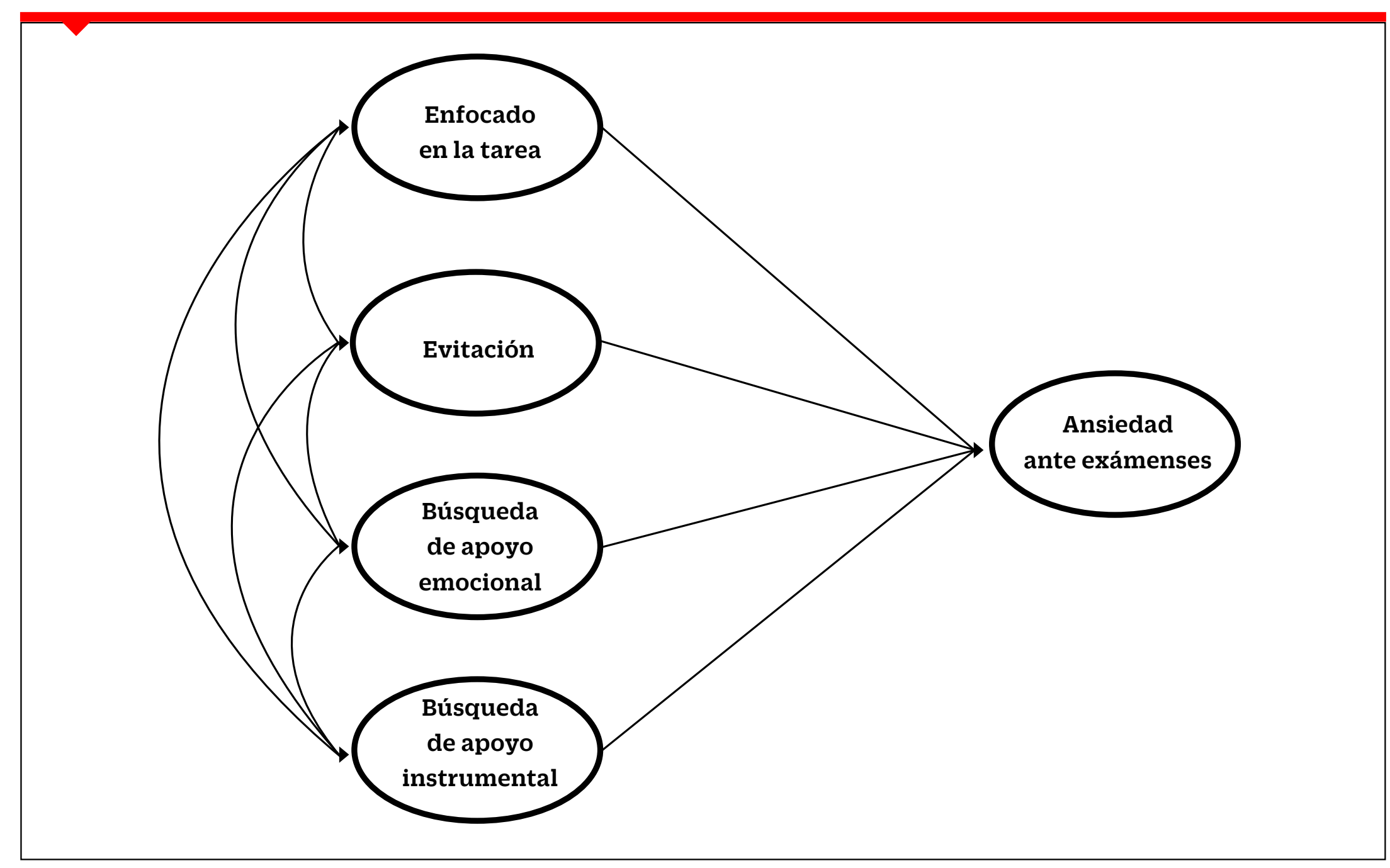

Figura 1. Modelo estructural.

Nota: las variables observadas (ítems) fueron omitidas para simplificar el gráfico.

El modelo fue valorado estadísticamente en función de dos criterios: suficiencia de los índices de ajuste y medidas de significancia práctica o magnitud del efecto (ME). Para el primer punto, fueron utilizados los índices de ajuste ya mencionados en párrafos anteriores (CFI, RMSEA y SRMR). Con relación a la ME, esta fue evaluada por medio del coeficiente de determinación $\left(R^{2}\right)$, es decir, el monto de variabilidad de la variable endógena atribuida a las variables exógenas, la influencia de cada variable exógena (coeficiente $B$ ) y su ME por medio de la $f^{2}$, al excluir dicha variable del modelo y recalcular el $R^{2}$ a fin de evaluar si el retiro de esa variable exógena afecta sustancialmente la varianza explicada del modelo (Cohen, 1988):

$$
f^{2}=\frac{R_{\text {incluido }}^{2}-R^{2} \text { excluido }}{1-R_{\text {incluido }}^{2}}
$$


En cuanto a la valoración de las medidas de ME, se considera al $R^{2}$ como significativo con un límite inferior de su intervalo de confianza (IC; Dominguez-Lara, 2017b) es $\geq .04$, y si $\beta>.20$ (Ferguson, 2009), respectivamente. Asimismo, en cuanto a la importancia de cada predictor, si $f^{2}>.02$ (Cohen, 1988) en el contexto de la investigación en ciencias sociales, indicaría que la influencia de cada variable exógena sobre la variable endógena es significativa. No fue considerada la significancia estadística en vista de que sus indicadores se hallan influidos sustancialmente por el tamaño muestral, e incluso en presencia de resultados estadísticamente no significativos, pueden hallarse ME relevantes, y viceversa (Kline, 2016).

\section{RESULTADOS}

Análisis descriptivo preliminar. La comparación realizada entre los coeficientes a de varones y mujeres indica que la diferencia no fue estadísticamente significativa excepto en $B A S$ instrumental, donde las mujeres presentaron indicadores más elevados (ver Tabla 1)

\section{Tabla 1}

Comparación de coeficientes entre varones y mujeres

\begin{tabular}{|c|c|c|c|c|c|c|}
\hline & & $\alpha$ & $\alpha$ AJUSTADO & W CORREGIDO & gl & $p$ \\
\hline \multirow{2}{*}{$\begin{array}{l}\text { Ansiedad ante } \\
\text { exámenes }\end{array}$} & Varones & .919 & .923 & 1.025 & 94,37 & .480 \\
\hline & Mujeres & .920 & .921 & & & \\
\hline \multirow{2}{*}{$\begin{array}{l}\text { Búsqueda de apoyo } \\
\text { emocional }\end{array}$} & Varones & .793 & .804 & 1.436 & 46,33 & .139 \\
\hline & Mujeres & .861 & .863 & & & \\
\hline \multirow{2}{*}{ Evitación } & Varones & .350 & .384 & 1.458 & 46,33 & .130 \\
\hline & Mujeres & .570 & .578 & & & \\
\hline \multirow{2}{*}{$\begin{array}{l}\text { Búsqueda de apoyo } \\
\text { instrumental }\end{array}$} & Varones & .669 & .686 & 1.773 & 46,33 & .044 \\
\hline & Mujeres & .820 & .823 & & & \\
\hline \multirow[b]{2}{*}{ Orientación a la tarea } & Varones & .766 & .778 & 1.200 & 46,33 & .294 \\
\hline & Mujeres & .812 & .815 & & & \\
\hline
\end{tabular}

Existe un predominio de las opciones más bajas en todas las dimensiones, excepto la estrategia Orientación a la tarea, donde predominan las respuestas más altas en la escala de respuesta (ver Tabla 2). Asimismo, algunos ítems del TAI-Estado presentan exceso de asimetría y curtosis (e.g., ítem 10), mientras que los ítems del COPEAU-B se mantienen dentro de límites aceptables (+/- 1.5; Pérez \& Medrano, 2010). En general, la prueba de SW ejecutada sugiere que la distribución de cada variable no se aproxima a la normalidad univariada $(\mathrm{p}<.001)$. 
Tabla 2

Estadísticos descriptivos de los ítems del TAI-Estado y COPEAU-B

\begin{tabular}{|c|c|c|c|c|}
\hline & $\mathbf{M}$ & $\mathrm{DE}$ & $\mathbf{g}^{1}$ & $\mathbf{g}^{2}$ \\
\hline \multicolumn{5}{|l|}{ Ansiedad ante exámenes } \\
\hline TAI1 & 2.147 & .708 & .778 & 1.059 \\
\hline TAI2 & 2.135 & .828 & .504 & -.117 \\
\hline TAI3 & 1.468 & .731 & 1.718 & 2.867 \\
\hline TAI 4 & 1.712 & .894 & 1.151 & .497 \\
\hline TAI5 & 1.705 & .738 & 1.019 & 1.161 \\
\hline TAI 6 & 2.058 & .781 & .640 & .377 \\
\hline TAI 7 & 1.538 & .666 & .853 & -.395 \\
\hline TAI 8 & 1.750 & .678 & .606 & .323 \\
\hline TAI 9 & 1.910 & .739 & .629 & .438 \\
\hline TAI 10 & 1.333 & .675 & 2.283 & 5.186 \\
\hline TAI 11 & 1.263 & .591 & 2.508 & 6.492 \\
\hline TAI 12 & 1.404 & .660 & 1.519 & 1.555 \\
\hline TAI13 & 1.910 & .868 & .835 & .184 \\
\hline TAI14 & 1.558 & .772 & 1.379 & 1.471 \\
\hline TAI 15 & 2.013 & .880 & .723 & -.014 \\
\hline \multicolumn{5}{|c|}{ Búsqueda de apoyo emocional } \\
\hline COPEAU1 & 2.551 & 1.271 & .857 & .452 \\
\hline COPEAU4 & 2.397 & 1.216 & .939 & .853 \\
\hline COPEAU5 & 2.628 & 1.316 & 1.025 & .635 \\
\hline \multicolumn{5}{|l|}{ Evitación } \\
\hline COPEAU 2 & 2.827 & 1.230 & .779 & .257 \\
\hline COPEAU 6 & 2.840 & 1.370 & .416 & -.603 \\
\hline COPEAU 10 & 2.782 & 1.143 & .569 & .385 \\
\hline \multicolumn{5}{|c|}{ Búsqueda de apoyo instrumental } \\
\hline COPEAU 3 & 2.314 & 1.353 & .853 & -.223 \\
\hline COPEAU 7 & 2.564 & 1.359 & .925 & .296 \\
\hline COPEAU 8 & 3.199 & 1.365 & .174 & -.709 \\
\hline \multicolumn{5}{|l|}{ Orientación a la tarea } \\
\hline COPEAU 9 & 3.513 & 1.257 & .158 & -.755 \\
\hline COPEAU 11 & 4.045 & 1.251 & -.146 & -.904 \\
\hline COPEAU 12 & 3.474 & 1.236 & .194 & -.562 \\
\hline
\end{tabular}

Nota: M: Media aritmética; DE: Desviación estándar; $g_{1}$ : Asimetría; $g_{2}$ : Curtosis 
Análisis de modelos de medición. El coeficiente de Mardia está por encima de lo considerado como adecuado (91.649), por lo que fue pertinente incluir una corrección de las estimaciones en ausencia de la normalidad.

Entre los modelos planteados, el que tuvo peor ajuste fue $\mathrm{M}_{1}$ (ver Tabla 3). Por el contrario, el modelo de medición que asocia las cuatro estrategias y la ansiedad ante exámenes (M2) obtuvo un ajuste favorable.

Con respecto a la validez convergente, el $\mathrm{M}_{2}$ mostró cargas factoriales de magnitud moderada $(\lambda>.50$; ver Tabla 4$)$, una varianza extraída promedio por factor aceptable (AVE > .50 ), excepto para Evitación ( $\mathrm{AVE}<.40$ ), así como correlaciones interfactoriales significativas (Tabla 5). La validez discriminante fue evaluada al comparar el cuadrado de las correlaciones interfactoriales y la AVE, encontrando que los factores se encuentran diferenciados empíricamente (ver Tabla 5). Con relación a la confiabilidad del constructo, la magnitud de los coeficientes fue elevada en la mayoría de los casos (> .70), excepto en Evitación (<.70). Entonces, con base en la evidencia mostrada, es pertinente continuar con el análisis estructural.

\section{Tabla 3}

Índices de ajuste de los modelos de medición

\begin{tabular}{|lrrrrrr|}
\hline \multicolumn{1}{|c}{ MODELO } & SB- $\boldsymbol{\chi}^{2}$ & $\mathbf{g l}$ & $\boldsymbol{p}$ & CFI & RMSEA (CI 90\%) & SRMR \\
\hline Modelos de medición & & & & & & \\
$\mathrm{M}_{1}$ & 895.252 & 323 & $<.001$ & .875 & $.107(.098-.115)$ & .135 \\
$\mathrm{M}_{2}$ & 433.823 & 314 & $<.001$ & .974 & $.050(.038-.060)$ & .071 \\
Modelo de regresión estructural & 420.103 & 304 & $<.001$ & .975 & $.050(.037-.061)$ & .071 \\
$\mathrm{M}_{3}$ & & & & & & \\
\hline
\end{tabular}

Nota: $M_{1}$ : Modelo unidimensional; $M_{2}:$ Modelo oblicuo; $M_{3}:$ Modelo estructural 
Tabla 4

Cargas factoriales de los ítems

\begin{tabular}{|c|c|c|c|c|c|}
\hline & ANS & BUSEMO & EVIT & BUSINST & TAREA \\
\hline TAI 1 & .584 & & & & \\
\hline TAI 2 & .577 & & & & \\
\hline TAI 3 & .760 & & & & \\
\hline TAI 4 & .598 & & & & \\
\hline TAI 5 & .690 & & & & \\
\hline TAI 6 & .705 & & & & \\
\hline TAI 7 & .720 & & & & \\
\hline TAI 8 & .710 & & & & \\
\hline TAI 9 & .732 & & & & \\
\hline TAI 10 & .827 & & & & \\
\hline TAI 11 & .826 & & & & \\
\hline TAI 12 & .751 & & & & \\
\hline TAI 13 & .684 & & & & \\
\hline TAI 14 & .799 & & & & \\
\hline TAI 15 & .772 & & & & \\
\hline COPEAU 1 & & .739 & & & \\
\hline COPEAU 4 & & .779 & & & \\
\hline COPEAU 5 & & .843 & & & \\
\hline COPEAU 2 & & & .592 & & \\
\hline COPEAU 6 & & & .709 & & \\
\hline COPEAU 10 & & & .350 & & \\
\hline COPEAU 3 & & & & .717 & \\
\hline COPEAU 7 & & & & .882 & \\
\hline COPEAU 8 & & & & .653 & \\
\hline COPEAU 9 & & & & & .706 \\
\hline COPEAU 11 & & & & & .810 \\
\hline COPEAU 12 & & & & & .706 \\
\hline
\end{tabular}

Nota: ANS: Ansiedad ante exámenes; BUSEMO: Búsqueda de apoyo emocional; EVIT: Evitación; BUSINST: Búsqueda de apoyo instrumental; TAREA: Orientación a la tarea 
Tabla 5

Evidencia convergente, discriminante, y confiabilidad del constructo

\begin{tabular}{|c|c|c|c|c|c|c|c|c|}
\hline & $\omega$ & $\mathbf{H}$ & AVE & ANS & BUSEMO & EVIT & BUSINST & TAREA \\
\hline ANS & .941 & .947 & .518 & & .077 & .051 & .108 & .023 \\
\hline BUSEMO & .830 & .839 & .621 & .277 & & .231 & .684 & .037 \\
\hline EVIT & .574 & .628 & .325 & .226 & .481 & & .275 & .004 \\
\hline BUSINST & .798 & .841 & .573 & .329 & .827 & .524 & & .030 \\
\hline TAREA & .786 & .796 & .551 & -.150 & .193 & -.063 & .172 & \\
\hline
\end{tabular}

Nota: ANS: Ansiedad ante exámenes; BUSEMO: Búsqueda de apoyo emocional; EVIT: Evitación; BUSINST: Búsqueda de apoyo instrumental; TAREA: Orientación a la tarea. AVE: varianza extraída promedio; Bajo la diagonal se encuentran las correlaciones; sobre la diagonal se halla la varianza compartida entre factores.

Análisis del modelo estructural. El modelo estructural (ver Figura 2) mostró índices de ajuste favorables (Tabla 3). Del mismo modo, las cuatro estrategias pre-examen explican de forma conjunta el 15.5\% de la variabilidad de la ansiedad ante exámenes $\left(R^{2}=.155\right.$; IC95\%.053 - .257; ver Figura 2), luego de corregir el coeficiente según el tamaño muestral y número de predictores (Kline, 2016), su magnitud no disminuyó notablemente $\left(R^{2}=.133\right.$; IC95\% .036 - .230).

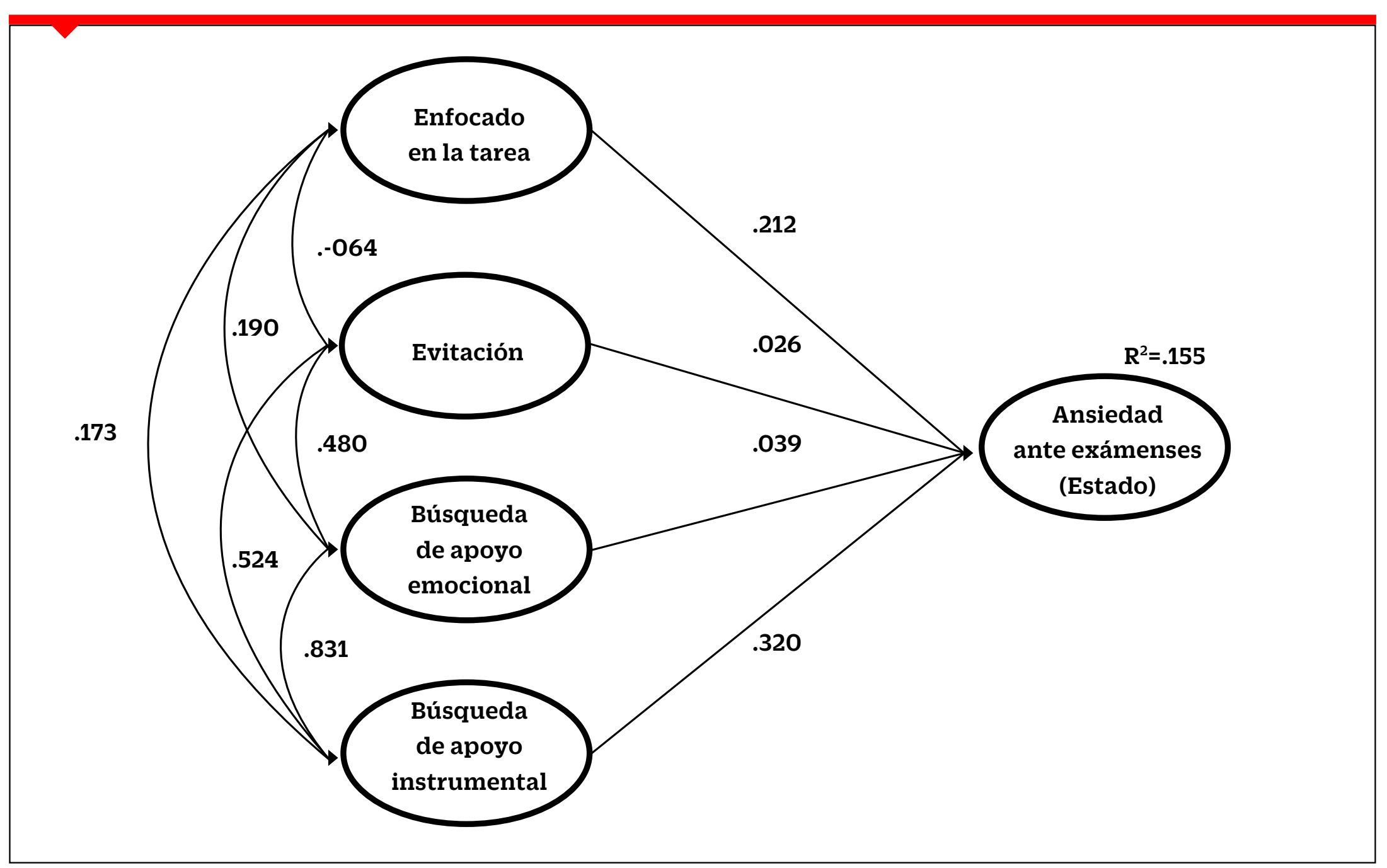

Figura 2. Modelo estructural con coeficientes estandarizados 
Finalmente, en cuanto al cumplimiento de las hipótesis, solo los coeficientes $B$ de las estrategias orientación a la tarea y búsqueda de apoyo instrumental son significativos (> .20; Ferguson, 2009), mostrando medidas de ME aceptables ( $\left.f^{2}>.02\right)$, lo que apoya la $\mathrm{H}_{1}$ y $\mathrm{H}_{2}$, de forma completa y parcial, respectivamente. Asimismo, la estrategia de evitación evidenció un efecto nulo (ver Tabla 6), lo que respalda $\mathrm{H}_{3}$.

\section{Tabla 6}

Resumen de la comprobación de las hipótesis de investigación

\begin{tabular}{|lccccc|}
\multicolumn{1}{c}{ RELACIÓN PROPUESTA } & TIPO DE EFECTO & $\boldsymbol{\beta}$ & $\mathbf{f}^{\mathbf{2}}$ & DECISIÓN \\
\hline Hipótesis 1 & TAREA $(-) \rightarrow$ ANS & Efecto directo & -.212 & .051 & Respaldada \\
Hipótesis 2 & BUSEMO $(+) \rightarrow$ ANS & Efecto directo & .039 & .021 & No respaldada \\
& BUSINST $(+) \rightarrow$ ANS & Efecto directo & .320 & .043 & Respaldada \\
Hipótesis 3 & EVIT(nulo) $\rightarrow$ ANS & Sin efecto & .026 & .001 & Respaldada \\
\hline
\end{tabular}

Nota: ANS: Ansiedad ante exámenes; BUSEMO: Búsqueda de apoyo emocional; EVIT: Evitación; BUSINST: Búsqueda de apoyo instrumental; TAREA: Orientación a la tarea; $\beta$ : coeficiente beta estandarizado; f2: medida de magnitud del efecto.

\section{DISCUSIÓN}

Tal como fue planteado hace más de seis décadas (Cronbach, \& Meehl, 1955), la red nomológica a la que pertenece determinado constructo es una fuente confiable para obtener evidencias de validez. Por ello, además del estudio de la estructura interna del TAI-Estado es necesario analizar si el constructo que evalúa se inserta apropiadamente dentro de dicha red. En este sentido, y de acuerdo con los estudios preliminares, las hipótesis de investigación estuvieron orientadas al análisis de la influencia de las EAPE sobre la experiencia de AE-Estado dentro del marco de las evidencias de validez con relación a otras variables, obteniendo evidencia favorable para dos de las hipótesis de forma completa, y una de forma parcial.

Los resultados obtenidos son consistentes con los hallazgos previos de la asociación de las EAPE y la AE como rasgo, sobre todo en lo concerniente a su relación con las estrategias orientadas a la BAS (Piemontesi \& Heredia, 2011; Putwain et al., 2012; Stöber, 2004), aunque en el presente estudio se observó una relación más consistente con la estrategia de BASinstrumental. Esto indica que los estudiantes que solicitan apoyo a sus compañeros sobre la forma de abordar el examen (BAS-instrumental) presentarían mayor AE, probablemente, por la inseguridad que les puede ocasionar una situación novedosa y no tener las herramientas necesarias para enfrentarlas eficientemente.

Por otro lado, la evidencia previa aparentemente contradictoria en cuanto a la relación entre las estrategias OT y E con la AE (Piemontesi \& Heredia, 2011; Putwain et al., 2012; Stöber, 
2004) pudo estar basada en mayor grado en las características de la muestra (e.g., estudiantes de secundaria en el estudio de Putwain et al. (2012), y universitarios en los otros dos trabajos) que repercutieron de algún modo en los resultados, o a la falta de claridad respecto de si las respuestas a los cuestionarios fueron brindadas de forma general o con relación a alguna asignatura en particular (Piemontesi \& Heredia, 2011; Stöber, 2004), ya que es importante considerar la influencia de la percepción de las asignaturas sobre la conducta académica del estudiante (Dominguez-Lara et al., 2017), incluyendo las EAPE.

De este modo, al menos con la muestra del presente estudio, aquellos estudiantes que se enfocan en el contenido de la asignatura dejando de lado diversos distractores antes del examen, experimentarían AE en menor grado. Esto tiene sentido porque un mayor conocimiento de los contenidos del curso otorgaría al estudiante un mayor grado de confianza, por lo que el examen no significaría una amenaza significativa. Sin embargo, es comprensible que la influencia no sea elevada porque incluso entre quienes se enfocan en los contenidos, usan una cantidad variable de estrategias que tienen diferentes grados de efectividad (Hong, Sas \& Sas, 2006).

Entre las implicancias prácticas de los hallazgos se destaca que estos ayudarán a los tutores universitarios a decidir sobre qué estrategias potenciar en los estudiantes (e.g., Orientación a la tarea) debido a su impacto negativo sobre la AE-Estado. Adicionalmente, las evidencias de validez acumuladas respecto al TAI-Estado posibilitan su inclusión dentro del sistema de evaluación que se realiza al estudiante, a fin de detectar a aquellos alumnos que muestren manifestaciones de ansiedad mayores y, por ende, vean afectado negativamente su rendimiento. Es necesario mencionar que esta evaluación debe complementarse con una medida de autorregulación académica y postergación de actividades, a fin de discernir entre una AE racional o irracional (Dominguez-Lara, 2016c).

En cuanto a los aspectos metodológicos del trabajo, resalta el uso de un procedimiento más potente que los análisis bivariados (Piemontesi \& Heredia, 2011; Stöber, 2004) o de regresión (Putwain et al., 2012) utilizados con antelación. No obstante, pese a que las interpretaciones derivadas de los hallazgos podrían cuestionarse a causa de la aparente debilidad de los resultados cuantitativos, las conclusiones sobre la validez de las inferencias no se basa únicamente en un resultado estadístico, sino en varias consideraciones, tanto empíricas como teóricas (Furr, 2011).

En primer lugar, es complicado obtener puntos de corte aceptables para valorar las medidas de $R^{2}$ encontradas (Hair, Ringle, \& Sarstedt, 2011) ya que dependen de la amplitud y complejidad de las variables estudiadas (Hair, Hult, Ringle, \& Sarstedt, 2014), por lo que se recurrió a planteamientos vinculados a las ciencias sociales $\left(R^{2}>.04\right.$; Ferguson, 2009) que permitan una valoración realista y menos restrictiva de los resultados hallados en el campo de la psicología. Esto es relevante porque en ocasiones los criterios procedentes de textos generales de estadística para valorar las ME no toman en cuenta las particularidades de cada disciplina (Gignac \& Szodorai, 2016). 
En segundo lugar, desde un plano teórico, a causa de la complejidad de la AE y los diversos factores involucrados en su génesis y mantenimiento, una varianza explicada por las EAPE alrededor del 15\% resulta significativa en este contexto de investigación, resaltando así el rol de dos de las estrategias: OT y BAS-instrumental. Cabe precisar que debido a la simplicidad del modelo, fue posible interpretar el $R^{2}$ de forma similar a un análisis de regresión (Prima, 2012).

Finalmente, no fueron realizadas re-especificaciones basadas en índices de modificación, ya que se busca contrastar el modelo teórico basado en antecedentes, mas no obtener un modelo a medida pero poco interpretable (Medrano \& Muñoz-Navarro, 2017), porque si bien los índices de ajuste estadístico en este marco analítico son importantes, no comandan las decisiones al momento de concluir sobre la validez de un modelo (Dominguez-Lara, 2016b).

En cuanto a las limitaciones del estudio destaca el tamaño muestral. Una de las exigencias naturales a los estudios que usan modelos de ecuaciones estructurales (MEE) es un tamaño de muestra elevado (Rusell, Kahn, Spoth, \& Altmaier, 1998), pero no es poco frecuente el uso de muestras pequeñas en estudios bajo esa metodología (Kline, 2016). Esta exigencia parece ser una condición necesaria para modelos complejos, con una gran cantidad de parámetros por estimar, aunque en modelos simples y con indicadores de elevada confiabilidad, podrían lograrse estimaciones adecuadas, incluso con muestras pequeñas (Hooper, Coughlan \& Mullen, 2008; Ullman, 2006), algunos problemas de estimación podrían persistir (Hoyle \& Gottfredson, 2015); pese a ello, si se cuenta con menos de 100 participantes, los resultados obtenidos a partir de un modelo planteado no serían confiables (Hu \& Bentler, 1995).

Otro aspecto por considerar es la potencial diferencia de las variables estudiadas con relación al sexo. A pesar de que pudo hallarse una equivalencia en cuanto a la estimación del error de medición mediante la comparación de coeficientes a que posibilitaría el tratamiento estadístico de la muestra de forma unitaria, esto no sustituye un análisis de invarianza que permitiría conocer más a fondo la igualdad de las relaciones entre los constructos y sus indicadores en cada uno de los grupos. Por tal motivo, los resultados podrían presentar variaciones en la relación entre las dimensiones estudiadas entre varones y mujeres. Cabe precisar que no se critica la proporción varones:mujeres (1:3), ya que es un panorama esperado en carreras como psicología, donde la mayoría son estudiantes de sexo femenino, y la institución en la que fueron evaluados los estudiantes no es la excepción.

Por último, las magnitudes de los coeficientes de confiabilidad fueron moderadas en la mayoría de los casos, excepto por evitación, que obtuvo coeficientes bajos ( $a, \omega$ y H) incluso para los estándares aceptados en investigación (<.70). Esto podría deberse a la cantidad de varones y las potenciales fluctuaciones al interior de ese grupo ( $n=39$ ), aunque el comportamiento psicométrico de dicha estrategia no ha variado con respecto a lo observado en investigaciones preliminares (Dominguez-Lara, 2018; Dominguez-Lara \& Merino-Soto, 2016) por lo que podría considerarse como algo previsible, y no propio de las características del grupo participante. 
En cuanto a las recomendaciones es necesario destacar algunos puntos. Es probable que la limitación referida a la cantidad de participantes no afecte la significancia práctica del modelo estructural (EAPE $\rightarrow$ AE) debido a la homogeneidad de la misma (estudiantes de psicología), la focalización en las asignaturas, y los indicadores de confiabilidad adecuados en la mayoría de las dimensiones evaluadas. Con todo, es recomendable que estos resultados sean replicados con una muestra de mayor tamaño a fin de fortalecer las conclusiones.

Asimismo, las características de la muestra podrían limitar la capacidad de generalización de los hallazgos para el investigador interesado en el tema, pero el objetivo del proyecto general en el cual se inserta el presente estudio fue conocer cómo se relacionan ambos constructos en un grupo determinado de estudiantes de psicología, mas no en estudiantes universitarios en general. Por ello, se recomienda replicar el análisis en estudiantes de otras profesiones a fin de enriquecer la discusión poniendo en relieve las diferencias existentes entre los estudiantes de diversas carreras profesionales.

Por otro lado, sería conveniente analizar la invarianza del modelo entre varones y mujeres, dado que fue un punto pendiente y podría brindar resultados enriquecedores, ya que las correlaciones entre las EAPE y la AE-Rasgo en un estudio previo mostraron diferencias según género (Stöber, 2004).

Para concluir, al ser la evaluación de la validez un proceso continuo (AERA, APA \& NCME, 2014), la implementación exitosa de una sola estrategia (e.g., evidencia basada en la estructura interna) no es determinante para justificar plenamente el uso de determinado instrumento en un contexto particular, por lo que es deseable obtener más de una evidencia de validez (Kline, 2005). En este sentido, los resultados del presente estudio aportan significativamente a la evidencia obtenida con respecto a la estructura interna del TAI-Estado (Dominguez-Lara, 2016a), así como las relaciones obtenidas con el rendimiento en exámenes (Dominguez-Lara, 2017a; Dominguez-Lara et al., 2017), y la consolidan como una medida adecuada de la AE durante la situación de examen. 


\section{REFERENCIAS}

American Educational Research Association, American Psychological Association, \& National Council on Measurement in Education (2014). Standards for educational and psychological testing. Washington, DC: American Educational Research Association.

Ato, M., López, J., \& Benavente, A. (2013). Un sistema de clasificación de los diseños de investigación en psicología. Anales de Psicología, 29(3), 1038-1059. doi: https://doi.org/10.6018/analesps.29.3.178511

Cohen, J. (1988). Statistical Power Analysis for Behavioural Sciences. New Jersey: Erlbaum.

Carver, C., \& Scheier, M. (1994). Situational coping and coping dispositions in a stressful encounter. Journal of Personality and Social Psychology, 66(1), 184-195. doi: https://doi.org/10.1037/0022-3514.66.1.184

Cronbach, L. \& Meehl, P. (1955). Construct validity in psychological tests. Psychological Bulletin, 52, 281-302. doi: https://doi.org/10.1037/h0040957

Dimitrov, D. M. (2010). Testing for factorial invariance in the context of construct validation. Measurement and Evaluation in Counseling and Development, 43(2), 121-149. doi: https://doi.org/10.1177/0748175610373459

Dominguez-Lara, S. (2016a). Inventario de la Ansiedad ante Exámenes-Estado: análisis preliminar de validez y confiabilidad en universitarios de Lima. Liberabit, 22(2), 219 - 228. Recuperado de https://goo.gl/4THm6z

Dominguez-Lara, S. (2016b). Evaluación de modelos estructurales, más allá de los índices de ajuste. Enfermería Intensiva, 27, 84-85. doi: https://doi.org/10.1016/j.enfi.2016.03.003

Dominguez-Lara, S. (2016c). Datos normativos de la Escala de Procrastinación Académica en estudiantes de psicología de Lima. Evaluar, 16, 20 - 30. Recuperado de https://goo.gl/uAraHy

Dominguez-Lara, S. (2017a). ¿Rendimiento en exámenes o promedio general? Algunas cuestiones sobre la medición del rendimiento académico en investigación. Pensando Psicología, 13(21), 33-39. doi: https://doi.org/10.16925/pe.v13i21.1712

Dominguez-Lara, S. (2017b). Magnitud del efecto en análisis de regresión. Interacciones, 3(1), 3-5. doi: https://doi. org/10.24016/2017.v3n1.46

Dominguez-Lara, S. (2018). Afrontamiento ante la ansiedad pre-examen y autoeficacia académica en estudiantes de ciencias de la salud. Educación Médica, 19(1), 39-42. doi: https://doi.org/10.1016/j.edumed.2016.07.007

Dominguez-Lara, S. \& De la Cruz-Contreras, F. (2017). Análisis estructural y desarrollo de una versión breve de la versión en español del Inventario de Ansiedad ante Exámenes (TAI-E) en universitarios de Lima. Interacciones, 3(1), 7-17. doi: https://doi.org/10.24016/2017.v3n1.50

Dominguez-Lara, S. \& Merino-Soto, C. (2016). Análisis Estructural de la Escala de Afrontamiento ante la Ansiedad e Incertidumbre Pre-examen (COPEAU) en universitarios peruanos. Revista Digital de Investigación en Docencia Universitaria, 10(2), 32 - 47. doi: https://doi.org/10.19083/ridu.10.474

Dominguez-Lara, S. \& Merino-Soto, C. (2017). Versión breve de la Escala de Afrontamiento ante la Ansiedad e Incertidumbre Pre-examen (COPEAU) en universitarios peruanos. Educación Médica 19(2), 67-72. doi: https://doi. org/10.1016/j.edumed.2017.04.011

Dominguez-Lara, S., Calderón-De la Cruz, G., Alarcón-Parco, D. \& Navarro-Loli, J. S. (2017). Relación entre Ansiedad ante exámenes y rendimiento en exámenes en universitarios: análisis preliminar de la diferencia según asignatura. Revista Digital de Investigación en Docencia Universitaria, 11(1), 166 - 176. doi: https://doi.org/10.19083/ridu.11.492

Feldt, L. S., \& Kim, S. (2006). Testing the difference between two alpha coefficients with small samples of subjects and raters. Educational and Psychological Measurement, 66(4), 589-600. doi: https://doi.org/10.1177/0013164405282488

Ferguson, C. J. (2009). An effect size primer: a guide for clinicians and researchers. Professional Psychology: Research and Practice, 40(5), 532-538. doi: https://doi.org/10.1037/a0015808

Fernández-Castillo, A., \& Caurcel, M.J. (2015). State test-anxiety, selective attention and concentration in university students. International Journal of Psychology, 50(4), 265-271. doi: https://doi.org/10.1002/ijop.12092

Fornell, C., \& Larcker, D. F. (1981). Evaluating structural equation models with unobservable variables and measure- 
ment error. Journal of Marketing Research, 18(1), 39- 50. doi: https://doi.org/10.2307/3151312

Furr, R.M. (2011). Scale construction and psychometrics for social and personality psychology. London: Sage publications. Ghasemi, A., \& Zahediasl, S. (2012). Normality tests for statistical analysis: a guide for non-statisticians. International Journal of Endocrinology and Metabolism, 10(2), 486-489. doi: https://doi.org/10.5812/ijem.3505

Gignac, G., \& Szodorai, E.T. (2016). Effect size guidelines for individual differences researchers. Personality and Individual Differences, 102, 74-78. doi: https://doi.org/10.1016/j.paid.2016.06.069

Hair, J. F., Ringle, C. M., \& Sarstedt, M. (2011). PLS-SEM: indeed a silver bullet. Journal of Marketing Theory and Practice, 19(2), 139-151. doi: https://doi.org/10.2753/MTP1069-6679190202

Hair, J. F., Hult, G.T.M., Ringle, C. M., \& Sarstedt, M. (2014). A primer on partial least squares structural equation modeling (Pls-Sem). California: Sage Publications.

Hair, J. F., Black, B., Babin, B., Anderson, R. E., \& Tatham, R. L. (2010). Multivariate data analysis. New York: Prentice Hall. Hancock, G. R., \& Mueller, R. O. (2001). Rethinking construct reliability within latent variable systems. En R. Cudeck, S. H. C. du Toit \& D. Sörbom (Eds.), Structural equation modeling: Past and present. A Festschrift in honor of Karl G. Jöreskog (pp. 195-261). Chicago: Scientific Software International.

Hong, E. (1998). Differential stability of individual differences in state and trait test anxiety. Learning and Individual Differences, 10(1), 51-69. doi: https://doi.org/10.1016/S1041-6080(99)80142-3

Hong, E., \& Karstersson, L. (2002). Antecedents of state test anxiety. Contemporary Educational Psychology, 27(2), 348 - 367. doi: https://doi.org/10.1006/ceps.2001.1095

Hong, E., Sas, M., \& Sas, J.C. (2006). Test-taking strategies of high and low mathematics achievers. Journal of Educational Research, 99(3), 144 - 155. doi: https://doi.org/10.3200/JOER.99.3.144-155

Hooper, D., Coughlan, J., \& Mullen, M.R. (2008). Structural equation modeling: guidelines for determining model fit. Journal of Business Research Methods, 6(1), 53 - 60. Recuperado de https://goo.gl/2VeKz6

Hoyle, R. H., \& Gottfredson, N. C. (2015). Sample size considerations in prevention research applications of multilevel modeling and structural equation modeling. Prevention Science, 16(7), 987 - 996. doi: https://doi. org/10.1007/s11121-014-0489-8

Hu, L., \& Bentler, P.M. (1995). Evaluating model fit. En R.H. Hoyle (Ed.). Structural equation modeling: Concepts, issues, and applications (pp. 76 - 99). California: Sage publications.

Ikeda, M., Iwanaga, M., \& Seiwa, H. (1996). Test anxiety and working memory system. Perceptual and Motor Skills, 82(3), 1223-1231. doi: https://doi.org/10.2466/pms.1996.82.3c.1223

Kline, R.B. (2016). Principles and practice of structural equation modeling. New York: The Guilford Press.

Kline, T. J. B. (2005). Psychological Testing: A Practical Approach to Design and Evaluation. California: Sage Publications.

Lee, S.-Y., Poon, W.-Y., \& Bentler, P. M. (1995). A two-stage estimation of structural equation models with continuous and polytomous variables. British Journal of Mathematical and Statistical Psychology, 48(2), 339-358. doi: https://doi.org/10.1111/j.2044-8317.1995.tb01067.x

Lotz, C., \& Sparfeldt, J.R. (2017). Does test anxiety increase as the exam draws near? - Students' state test anxiety recorded over the course of one semester. Personality and Individual Differences, 104, 397-400. doi: https:// doi.org/10.1016/j.paid.2016.08.032

McDonald R.P. (1999). Test theory: A unified treatment. Mahwah, N.J.: L. Erlbaum Associates.

Medrano, L. \& Muñoz-Navarro, R. (2017). Aproximación Conceptual y Práctica a los Modelos de Ecuaciones Estructurales. Revista Digital de Investigación en Docencia Universitaria, 11(1), 219 - 239. doi: https://doi.org/10.19083/ ridu.11.486

Merino-Soto, C. (2016). Diferencias entre coeficientes alfa de Cronbach, con muestras y partes pequeñas: Un programa VB. Anales de Psicología, 32(2), 587 - 588. doi: https://doi.org/10.6018/analesps.32.2.203841

Merino, C., \& Lautenschlager, G. (2003). Comparación estadística de la confiabilidad alfa de Cronbach: Aplicaciones en la medición educacional. Revista de Psicología - Universidad de Chile, 12, 129-139. Recuperado de https:// goo.gl/BAW9CV 
Pérez, E. \& Medrano, L. (2010). Análisis Factorial Exploratorio: Bases Conceptuales y Metodológicas. Revista Argentina de Ciencias del Comportamiento, 2(1), 58-66. Recuperado de https://goo.gl/EqqXjn

Piemontesi, S. \& Heredia, D. (2011). Relaciones entre la ansiedad frente a los exámenes, estrategias de afrontamiento, autoeficacia para el aprendizaje autorregulado y rendimiento académico. Revista Tesis, 1, 74 - 86. Recuperado de https://goo.gl/q1LhWa

Prima, B. (2012). Antecedents and consequences of female consumer expectations and evaluations toward CSR activities. Asean Marketing Journal, 4(1), 47 - 56. doi: https://doi.org/10.21002/amj.v4i1.2031

Putwain, D., Connors, L., Symes, W., \& Douglas-Osborn, E. (2012). Is academic buoyancy anything more tan adaptative coping? Anxiety, Stress, and Coping, 25(3), 349-358. doi: https://doi.org/10.1080/10615806.2011.582459

Rodríguez, M. \& Ruiz, M. (2008). Atenuación de la asimetría y de la curtosis de las puntuaciones observadas mediante transformaciones de variables: Incidencia sobre la estructura factorial. Psicológica, 29, 205-227. Recuperado de https://goo.gl/bgEBcH

Roick, J., \& Ringeisen, T. (2017). Self-efficacy, test anxiety, and academic success: A longitudinal validation. International Journal of Educational Research, 83, 84-93. doi: https://doi.org/10.1016/j.ijer.2016.12.006

Rusell, D.W., Kahn, J.H., Spoth, R., \& Altmaier, E.M. (1998). Analyzing data from experimental studies: a latent variable structural equation modeling approach. Journal of Counseling Psychology, 45(1), 18 - 29. doi: https:// doi.org/10.1037/0022-0167.45.1.18

Satorra, A., \& Bentler, P. M. (1994). Corrections to test statistics and standard errors in covariance structure analysis. En A. von Eye \& C. C. Clogg (Eds.), Latent variables analysis: Applications for developmental research (pp. 399-419). Thousand Oaks, CA: Sage.

Shapiro, S.S., \& Wilk, M.B. (1965). An Analysis of Variance Test for Normality (complete Samples). Biometrika, 52(3/4), 591-611. doi: https://doi.org/10.2307/2333709

Stöber, J. (2004), Dimensions of test anxiety: relations to ways of coping with pre-exam anxiety and uncertainty. Anxiety, Stress, and Coping, 17(3), 213-226. doi: https://doi.org/10.1080/10615800412331292615

Spielberger, C.D. (1980). Tensión y Ansiedad. México: Harla.

Spielberger, C. D., \& Vagg, P. (1995). Test Anxiety. A transactional process. En C. Spielberger \& P. Vagg (Eds.), Test Anxiety: Theory, assessment and treatment (pp. 3-14). Washington: Taylor \& Francis.

Taras, V., \& Kline, T. (2010). Scale validation via quantifying item validity using the Dm Index. Psychological Reports, 107(2), 535-546. doi: https://doi.org/10.2466/03.PR0.107.5.535-546

Ullman, J. B. (2006). Structural equation modeling: Reviewing the basics and moving forward. Journal of Personality Assessment, 87(1), 35 - 50. doi: https://doi.org/10.1207/s15327752jpa8701_03

Zohar, D. (1998). An additive model of test anxiety: Role of exam-specific expectations. Journal of Educational Psychology, 90(2), 330-340. doi: https://doi.org/10.1037/0022-0663.90.2.330

Nota de autor: Este artículo fue elaborado en el marco del proyecto "Modelo explicativo del rendimiento en exámenes escritos en estudiantes de psicología de la USMP: validación e intervención" del Instituto de Investigación de Psicología de la Universidad de San Martín de Porres (Lima, Perú).

RIDU / Revista Digital de Investigación en Docencia Universitaria / ISNN 2223-2516

(C) Los autores. Este artículo es publicado por la Revista Digital de Investigación en Docencia Universitaria del Área de Institutional Research and Effectiveness de la Dirección de Aseguramiento de la Calidad, Universidad Peruana de Ciencias Aplicadas. Este es un artículo de acceso abierto, distribuido bajo los términos de la LicenciaCreativeCommons Atribución-CompartirIgual 4.0 Internacional ( http://creativecommons.org/licenses/by-sa/4.0/), que permite el uso no comercial, distribución y reproducción en cualquier medio, siempre que la obra original sea debidamente citada. 